\title{
MEASUREMENTS OF A CRENELATED IRON POLE TIP FOR THE VLHC TRANSMISSION LINE MAGNET
}

\author{
J.DiMarco, G.W.Foster, V.Kashikhin ${ }^{+}$, A. Makarov, P.Schlabach \\ Fermi National Accelerator Laboratory*, Batavia, IL
}

\begin{abstract}
The Very Large Hadron Collider (VLHC) is under conceptual design in Fermilab. One option under development is a 2-Tesla warm iron 2-in-1 single turn superferric magnet built around an $80 \mathrm{kA}$ superconducting transmission line. A normalconducting test stand was built to optimize the iron lamination shape for this magnet. It uses a watercooled copper winding to provide the $100 \mathrm{kA}$-turns needed to generate 2 Tesla fields in both $20 \mathrm{~mm}$ air gaps of the magnet. A magnetic measurement facility has been set up for magnetic field mapping, which includes a flat measurement coil, precision stage for coil motion and integrator. Results from a first test of the "crenelation" technique to mitigate the saturation sextupole in iron magnets are described and future plans are discussed.
\end{abstract}

\section{INTRODUCTION}

Parameters of the VLHC Transmission Line Magnet [1] are given in Table 1.

\begin{tabular}{|c|c|}
\hline Magnet Type & Warm Iron Superferric \\
\hline Number of turns & 1 \\
\hline Beam gaps & 2-in-1 \\
\hline Drive Conductor & $\begin{array}{l}\text { 80-100kA Supercond. } \\
\text { Transmission Line }\end{array}$ \\
\hline Maximum field & 2 Tesla \\
\hline Injection field & 0.1 Tesla \\
\hline Gradient & $+/-3 \% / \mathrm{cm}$ \\
\hline Quadrupoles & None required \\
\hline Air gap height & $20 \mathrm{~mm}$ \\
\hline $\begin{array}{l}\text { Good field }(<0.01 \%) \\
\text { diameter: Injection } \\
2 \text { Tesla }\end{array}$ & $\begin{array}{l}20 \mathrm{~mm} \\
10 \mathrm{~mm}\end{array}$ \\
\hline Superconductor & NbTi or Nb3Al \\
\hline Magnetic field energy & 12 kJ/m @ 100kA \\
\hline
\end{tabular}

An important issue with this magnet design is the preservation of field quality as the iron approaches saturation at $\mathrm{B}=1.7 \sim 2.2 \mathrm{~T}$. In this superferric magnet the cost of providing the additional ampere-turns is low, in contrast to copper-driven magnets. However

\footnotetext{
*Operated by the URA, under contract with the U.S. DOE

+ email:Kash@fnal.gov
}

this advantage can only be realized if the field quality is preserved at high excitation, at least to the extent sufficient to preserve dynamic aperture for the small beam sizes at flat top. A number of techniques are available to control saturation effects in iron magnets. These include holes in the laminations in the regions above the pole tip and "crenelation" techniques [1,2]. Roughly speaking, all these methods work by reducing the average density of the iron near the middle of the pole tip so that the magnet saturates uniformly and field quality is preserved. Understanding and controlling these techniques will likely take many design iterations and a convenient test facility.

\section{TEST STAND}

The cryogenic and superconductor development program underway for this magnet is described in [3]. To optimize the warm iron lamination design it is expedient to use a normal conducting test stand on which variants of the iron shape can be tested without the complexities of a cryogenic system.

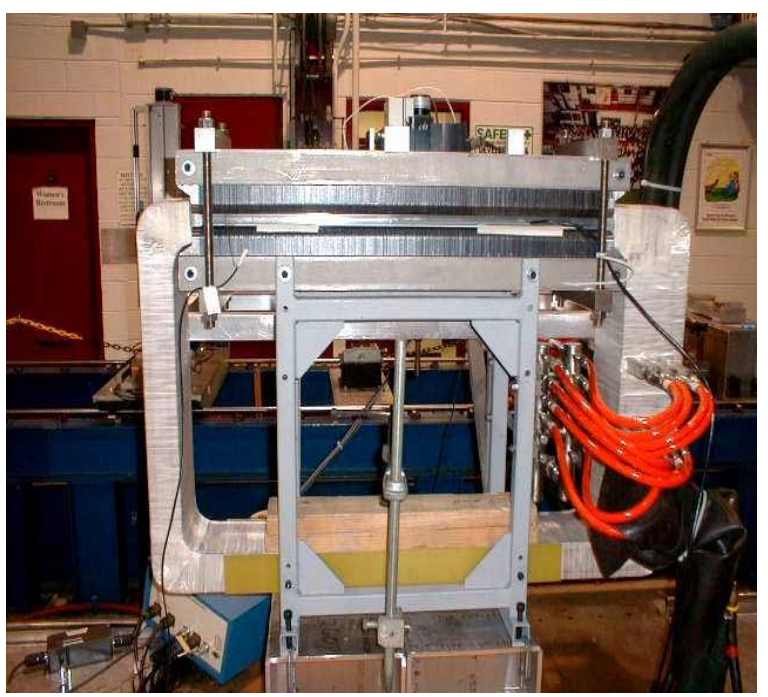

Fig. 1 - Transmission line magnet test stand including 1 meter long laminated steel cores and 100kA-turn copper coil with ten individual water circuits.

The test stand constructed at Fermilab to investigate various forms and designs of magnetic core for transmission line magnets is shown in fig. 1. The test requires $80-100 \mathrm{kA}$-turns to pass through the $8 \mathrm{~cm}$ hole in the center of the magnet that would normally be occupied by the superconducting transmission line. It is 
calculated that only small field shape differences will occur between the superconducting and normal conductors arising from the difference in their radial extents. This current density is an engineering challenge with water-cooled copper coils and required ten separate water circuits for each of the 10 turns of 10kA each. The coil was powered by the filtered and regulated 10kA supply originally used to test dipoles for the Fermilab Main Injector. The coil, magnet core, and probe station are mounted on an aluminum frame.

\section{MAGNET CORES}

The magnet cores were manufactured from $1.5 \mathrm{~mm}$ thick low carbon steel laser cut laminations. See fig. 2. Each core has two holes (not shown) for alignment pins which run the full length of the magnet. Two aluminum bars installed between cores fix the air gaps on both sides of the magnet. Four threaded rods on both sides of magnet clamp the upper and lower cores into one mechanical structure. This arrangement allows simple and quick assembly/disassembly of the magnet. Both magnet air gaps are open for field measurements.

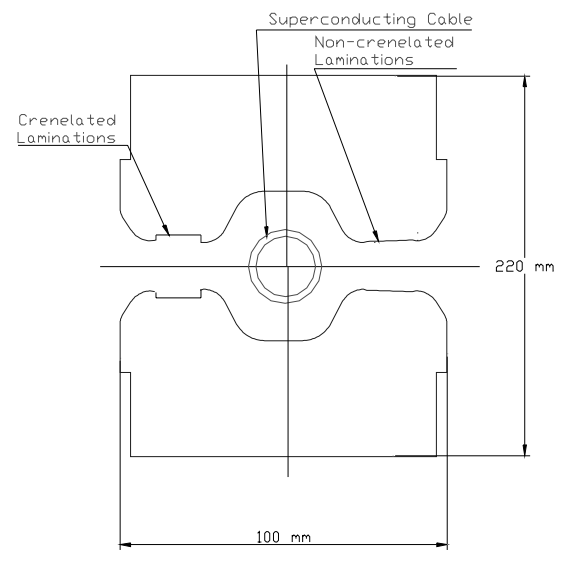

Fig. 2 - Lamination drawing of the steel cores showing position of the superconducting transmission line (or copper drive coil) and the two air gaps for the beams. In the crenelation technique every $\sim 10^{\text {th }}$ lamination has a cutout (shown in left gap on figure) which reduces the average iron density in the center of the pole tip to reduce the saturation sextupole.

\section{MAGNET MEASUREMENTS}

Field shape measurements were made with a flat coil glued to a G-10 plate. The coil had 3 turns, $2 \mathrm{~mm}$ width, and $800 \mathrm{~mm}$ length. The coil could be moved in horizontal and vertical directions by high precision (accuracy 1um) computer controlled stage. Field shape measurements were made using the integrated signal from the coil as it was moved into the aperture. The power supply was turned off at the start and end of each measurement in order to measure the zero point of the flux and cancel the effects of integrator drift.
A reference probe placed in the second magnet gap was used to normalize out the effects of power supply noise (mostly $720 \mathrm{~Hz}$ ). At low field levels (0.1-0.5T) power supply noise was still significant and a MathCad filtering technique was used to smooth the data.

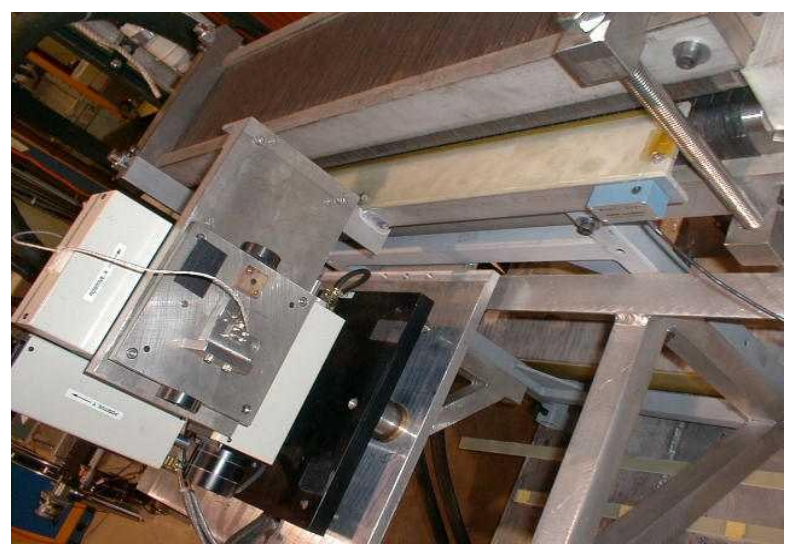

Fig.3 - Stage and Measuring Coil

\section{POLE TIP DESIGN ISSUES}

The main design problem in this type of magnet is to retain high magnetic field quality at the top end of the $0.1 \mathrm{~T}-2 \mathrm{~T}$ operating range. The saturation of the flux return regions of the iron cores can be controlled by increasing their thickness. However the pole tips are unavoidably highly saturated when the fields reach 2 Tesla. The magnetic permeability changes by about a factor of 1000 from injection to maximum field, and the saturation is most pronounced at the corners of the pole profiles which carry maximum flux. This reduces the gap flux disproportionately at the edges of the aperture at high excitation, leading to the "saturation sextupole" at high fields. In addition, gradient shifts can occur in combined-function or asymmetric designs.

A number of technical approaches have been tried reduce these effects. Most of them use active field correction by placing an additional winding into the air gap. It is possible also to split the main winding in window frame magnet and change the current ratio between the parts to reduce the sextupole component during acceleration cycle. This technique was used in the TAC design of a superferric magnet for the SSC.

The "Crenelation" technique was proposed in [1]. The main idea is to reduce the iron density in the pole center by machining small indentations ("crenelations") in each N-th lamination. See fig. 2. The height and the width of this slot should be optimized using computer codes. Such an attempt was made using 2-dimensional codes and described in $[4,5]$. However it should be noted that the flux redistribution around "crenelations" is a three-dimensional problem which is only approximated by the reduced average 
density ("stacking factor") of 2-dimensional codes. A direct solution to the 3D-magnetostatic problem is prohibitively difficult. Our goal in these measurements is to obtain an effective 2-D model for the crenelation which can be used for overall pole tip optimization.

\section{RESULTS}

For this investigation we used the simplified lamination configuration shown on Fig.2. Because the laminations were laser cut to an accuracy of only $\sim 0.003 "$, the field defect on the midplane was of order $0.1 \%$ at the design aperture. This was acceptable for this study since the field shape change could still be studied as a function of excitation and crenelation structure. Several variants of the pole "crenelations" were measured at currents up to $100 \mathrm{kA}$ and stacking factors Kc ranging between 0 and $17.7 \%$.

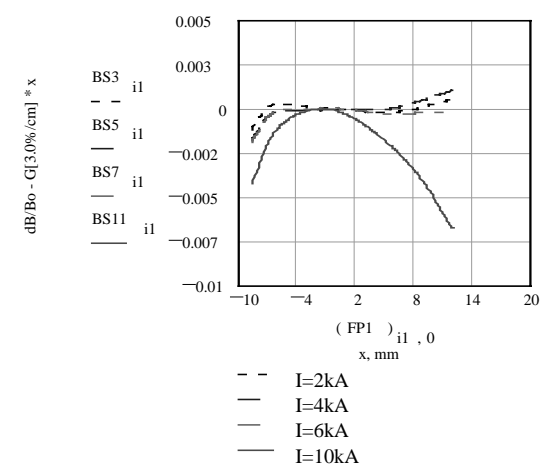

Fig.4 Field shape without crenelations.

In the pole tip without crenelations (fig.4) the field shape was stable between 0.1 and $1.7 \mathrm{~T}$ as expected. Above 1.7T the field developed a significant sextupole as well as a gradient shift arising from the asymmetry of the double-C magnet design.

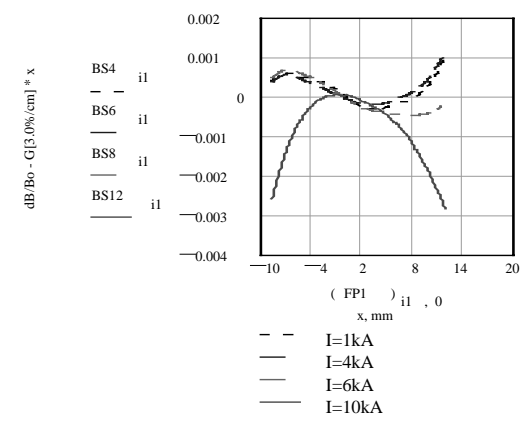

Fig.5 Field shape with crenelations, Kc=10\%.

With every $10^{\text {th }}$ lamination crenelated (fig. 5) two effects were observed. Firstly the saturation sextupole at high field was reduced as expected. Secondly (and unexpectedly) the low field shape changed by several parts in $10^{-4}$. This effect is not predicted by the 2-D models of the crenelation, which indicate that the field shape should be independent of the crenelations for $\mathrm{B}<1$ Tesla. Presumably this is due to $3-\mathrm{D}$ flux redistribution effects and/or the early onset of saturation in the exposed, sharp corners near the crenelations.

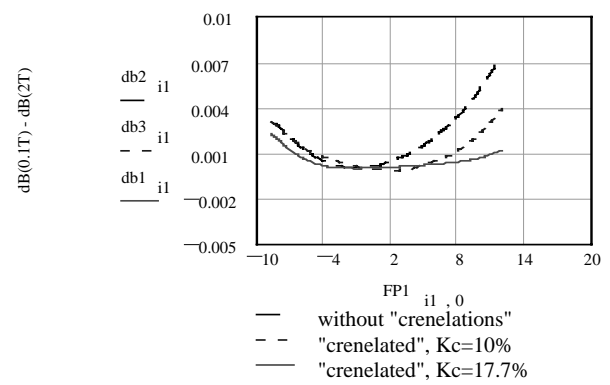

Fig.6 - Field shape change between $0.1 \mathrm{~T}$ and $2 \mathrm{~T}$ for no crenelations, $10 \%$ crenelation, and $17.7 \%$ crenelation. Positive curvature indicates negative sextupole at $2 \mathrm{~T}$.

The results are summarized in fig. 6, which shows the shape change between $0.1 \mathrm{~T}$ and $2 \mathrm{~T}$ for different crenelation stacking factors. The curves that even this simple crenelations causes significant reduction in field shape change due to saturation.

\section{CONCLUSIONS}

The field measurements confirmed the positive influence of crenelations on the field quality for highly saturated magnets. It seems plausible that an optimized crenelation will be able to substantially eliminate the saturation defect at 2 Tesla. An unexpected result is that crenelations also influence (at the level of level of a few parts in $10^{-4}$ ) the shape of the magnetic field at low excitation. This makes the crenelation technique less attractive to use since the low field pole tip shape and the design of the crenelation become coupled. The field correction by "holes in the poles" remains a promising approach which should avoid these difficulties.

\section{REFERENCES}

[1] R.R.Wilson Procceedings of Snowmass 1982.

[2] S. Snowdon, "Magnetic Considerations in a Superferric Dipole" FNAL-TM-1210 March 1983.

[3] G.W. Foster et al, these proceedings.

[4] G.W.Foster "Magnetic Calculations for the Transmission Line Magnet". Proceedings of Snowmass '96, January 1997.

[5] V.Kashikhin-Jr., G.W.Foster, V.Kashikhin, EPAC '98, Stockholm, 1998,p.1934 - 1936. 